\title{
Standard(-like) Model from an SO(12) Grand Unified Theory in six-dimensions with $S^{2}$ extra space
}

Takaaki Nomura*

Department of Physics, Saitama University, Shimo-Okubo, Sakura-ku, Saitama 355-8570, Japan

E-mail: nomura@krishna.th.phy.saitama-u.ac.jp

\section{Joe Sato}

Department of Physics, Saitama University, Shimo-Okubo, Sakura-ku, Saitama 355-8570, Japan

E-mail: joe@phy.saitama-u.ac.jp

\begin{abstract}
We analyze a gauge-Higgs unification model based on a gauge theory on a six-dimensional spacetime which has an $S^{2}$ extra-space. We impose a symmetry condition for a gauge field and nontrivial boundary conditions on the $S^{2}$ for each fields. We briefly review the scheme for constructing a four-dimensional theory from the six-dimensional gauge theory under these conditions. We then construct a specific model based on an $\mathrm{SO}(12)$ gauge theory with fermions which lie in a 32 representation of $\mathrm{SO}(12)$, under the scheme. We find that this model leads a StandardModel(-like) gauge theory which has gauge symmetry $\mathrm{SU}(3) \times \mathrm{SU}(2)_{L} \times \mathrm{U}(1)_{Y}\left(\times \mathrm{U}(1)^{2}\right)$ and one generation of SM fermions, in four-dimensions. The Higgs sector of the model is also analyzed, and it is shown that the electroweak symmetry breaking and the prediction of W-boson and Higgs-boson masses are obtained.
\end{abstract}

The 2009 Europhysics Conference on High Energy Physics,

July 16 - 222009

Krakow, Poland

\footnotetext{
* Speaker.
} 


\section{1. introduction}

The gauge-Higgs unification is one of the attractive approaches to the physics beyond the SM [1 2 3]. In this approach, the Higgs particles originate from the extra-dimensional components of the gauge field of a gauge theory defined on spacetime with dimensions larger than four. Thus the Higgs sector is embraced into the gauge interactions in the higher-dimensional spacetime and part of the fundamental properties of Higgs scalar is determined from the gauge interactions. We consider, in this paper, gauge-Higgs unification model on six-dimensional spacetime which has $S^{2}$ extra-space with non-trivial boundary conditions of fields on $S^{2}$.

\section{Model}

We consider a gauge-Higgs unification model based on a gauge theory as defined on the sixdimensional spacetime with the extra-space which has the structure of two-sphere $S^{2}$ [4]. We can impose on the fields of this gauge theory the symmetry condition which identifies the gauge transformation as the isometry transformation of $S^{2}$ as in the coset space dimensional reduction(CSDR) scheme, since the $S^{2}$ has the coset space structure such as $S^{2}=\mathrm{SU}(2) / \mathrm{U}(1)$. We then impose on the gauge field the symmetry in order to carry out the dimensional reduction of the gauge sector.

The action of this theory is given by

$$
S=\int d x^{4} \sin \theta d \theta d \phi\left(\bar{\psi} i \Gamma^{\mu} D_{\mu} \psi+\bar{\psi} i \Gamma^{a} e_{a}^{\alpha} D_{\alpha} \psi-\frac{1}{4 g^{2}} g^{M N} g^{K L} \operatorname{Tr}\left[F_{M K} F_{N L}\right]\right)
$$

where $F_{M N}=\partial_{M} A_{N}(X)-\partial_{N} A_{M}(X)-\left[A_{M}(X), A_{N}(X)\right]$ is the field strength, $D_{M}$ is the covariant derivative including spin connection, and $\Gamma_{A}$ represents the 6-dimensional Clifford algebra. We impose on the gauge field $A_{M}(X)$ the symmetry which connects $\mathrm{SU}(2)_{I}$ isometry transformation on $S^{2}$ and the gauge transformation on the fields in order to carry out dimensional reduction, and the non-trivial boundary conditions of $S^{2}$ to restrict four-dimensional theory. The symmetry requires that the $\mathrm{SU}(2)_{I}$ coordinate transformation should be compensated by a gauge transformation. The symmetry further leads to the following set of the symmetry condition on the fields [1, 5, 6 :

$$
\begin{aligned}
\xi_{i}^{\beta} \partial_{\beta} A_{\mu} & =\partial_{\alpha} W_{i}+\left[W_{i}, A_{\mu}\right], \\
\xi_{i}^{\beta} \partial_{\beta} A_{\alpha}+\partial_{\alpha} \xi_{i}^{\beta} A_{\beta} & =\partial_{\alpha} W_{i}+\left[W_{i}, A_{\alpha}\right],
\end{aligned}
$$

where $\xi_{i}^{\alpha}$ is the Killing vectors generating $\mathrm{SU}(2)_{I}$ symmetry and $W_{i}$ are some fields which generate an infitesimal gauge transformation of $G$. Here index $i=1,2,3$ corresponds to that of $\mathrm{SU}(2)$ generators. The LHSs of Eq (2.2/2.3) are infintesimal isometry $\mathrm{SU}(2)_{I}$ transformation and the RHSs of those are infintesimal gauge transformation. The non-trivial boundary conditions are 
defined so as to remain the action Eq (2.1) invariant, and are written as

$$
\begin{gathered}
\psi(x, \pi-\theta,-\phi)=\gamma_{5} P \psi(x, \theta, \phi), \\
A_{\mu}(x, \pi-\theta,-\phi)=P A_{\mu}(x, \theta, \phi) P, \\
A_{\theta}(x, \pi-\theta,-\phi)=-P A_{\theta}(x, \theta, \phi) P, \\
A_{\phi}(x, \pi-\theta,-\phi)=-P A_{\phi}(x, \theta, \phi) P, \\
\psi(x, \theta, \phi+2 \pi)=P^{\prime} \psi(x, \theta, \phi), \\
A_{\mu}(x, \theta, \phi+2 \pi)=P^{\prime} A_{\mu}(x, \theta, \phi) P^{\prime}, \\
A_{\theta}(x, \theta, \phi+2 \pi)=P^{\prime} A_{\theta}(x, \theta, \phi) P^{\prime}, \\
A_{\phi}(x, \theta, \phi+2 \pi)=P^{\prime} A_{\phi}(x, \theta, \phi) P^{\prime},
\end{gathered}
$$

where $P\left(P^{\prime}\right)$ s act on the representation space of gauge group $G$ and satisfy $P^{2}=1\left(\left(P^{\prime}\right)^{2}=1\right)$; we can take element of $P\left(P^{\prime}\right)$ as \pm 1 . The fermion sector of four-dimensional action is obtained by expanding fermions in normal modes of $S^{2}$ and then integrating $S^{2}$ coordinate in six-dimensional action. Thus, the fermions have massive KK modes which would be a candidate of dark matter. Generally, the KK modes do not have massless mode because of the positive curvature of $S^{2}$. The existence of the positive curvature is expressed as spin connection term of covariant derivative in six-dimensional Lagrangian. We, however, can show that the fermion components satisfying the following condition have massless mode:

$$
-i \Phi_{3} \psi=\frac{\Sigma_{3}}{2} \psi
$$

since spin connection term in Eq. (Dphi) is canceled by this condition.

We then construct a model based on a gauge group $G=\mathrm{SO}(12)$ and a representation $F=32$ of $\mathrm{SO}(12)$ for fermions. Our set up is as follows.

1. We assume that $\mathrm{U}(1)_{I}$ is embedded into $\mathrm{SO}(12)$ such as

$$
S O(12) \supset S O(10) \times U(1)_{I} \text {. }
$$

2. The parity assignment is written in 32 dimensional spinor basis of $\mathrm{SO}(12)$ such as

$$
\begin{aligned}
S O(12) & \supset S U(3) \times S U(2)_{L} \times U(1)_{Y} \times U(1)_{X} \times U(1)_{I} \\
32= & (3,2)^{(+-)}(1,-1,1)+(\overline{3}, 2)^{(+-)}(-1,1,-1) \\
& +(3,1)^{(--)}(4,1,-1)+(\overline{3}, 1)^{(--)}(-4,-1,1) \\
& +(3,1)^{(-+)}(-2,-3,-1)+(\overline{3}, 1)^{(-+)}(2,3,1) \\
& +(1,2)^{(++)}(3,-3,-1)+(1,2)^{(++)}(-3,3,1) \\
& +(1,1)^{(--)}(6,-1,1)+(1,1)^{(--)}(-6,1,-1) \\
& +(1,1)^{(-+)}(0,-5,1)+(1,1)^{(-+)}(0,5,-1),
\end{aligned}
$$

where e.g. $(+,-)$ means that the parities $\left(P, P^{\prime}\right)$ of the associated components are (even, odd).

3. We introduce two types of left-handed Weyl fermions that belong to 32 representation of $\mathrm{SO}(12)$, which have parity assignments such as $\psi^{\left(+P^{\prime}\right)} \rightarrow \gamma_{5} P \psi^{\left(+P^{\prime}\right)}\left(P^{\prime} \gamma_{5} \psi^{\left(+P^{\prime}\right)}\right)$ and $\psi^{\left(-P^{\prime}\right)}$ $\rightarrow \gamma_{5} P \psi^{\left(-P^{\prime}\right)}\left(-P^{\prime} \gamma_{5} \psi^{\left(-P^{\prime}\right)}\right)$ respectively. 


\section{The consequences of the model}

As a result of this set up, we obtain gauge symmetry breaking by symmetry condition and boundary condition as $\mathrm{SO}(12) \supset \mathrm{SO}(10) \times \mathrm{U}(1)_{I} \supset \mathrm{SU}(5) \times \mathrm{U}(1)_{X} \times \mathrm{U}(1)_{I} \supset \mathrm{SU}(3) \times \mathrm{SU}(2)_{L} \times$ $\mathrm{U}(1)_{Y} \times \mathrm{U}(1)_{X} \times \mathrm{U}(1)_{I}$, SM Higgs doublet $(1,2)(3,2,-2)$ and $(1,2)(-3,-2,2)$, and one generation of SM fermions $\left\{(3,2)(1,-1,1)_{L},(3,1)(4,1,-1)_{R},(3,1)(-2,-3,-1)_{R},(1,2)(-3,3,1)_{L},(1,1)(-6,1,-1)_{R},(1,1)(0,5,-\right.$ $\left.1)_{R}\right\}$.

We also analyzed Higgs potential and obtain vaccume expectation value of Higgs doublet as

$$
<\phi>=\frac{1}{\sqrt{2}}\left(\begin{array}{l}
0 \\
v
\end{array}\right), \quad v=\sqrt{\frac{4}{3}} \frac{1}{g R},
$$

and W boson mass $m_{W}$ and Higgs mass $m_{H}$ are given in terms of radius $R$

$$
m_{W}=g_{2} \frac{v}{2}=\sqrt{\frac{2}{3}} \frac{1}{R}, \quad m_{H}=\sqrt{3} g v=\sqrt{4} \frac{1}{R} .
$$

The ratio between $m_{W}$ and $m_{H}$ is predicted

$$
\frac{m_{H}}{m_{W}}=\sqrt{6}
$$

The electroweak symmetry breaking is then realized and the Higgs mass value is predicted.

\section{Summary}

We analyzed a gauge theory defined on the six-dimensional spacetime which has an $S^{2}$ extraspace, with the symmetry condition and non-trivial boundary conditions and constructed the model based on SO(12) gauge theory. We found that this model leads Standard Model like particle contents in four-dimensional spacetime and prediction for the Higgs sector.

\section{References}

[1] N.S. Manton, Nucl. Phys. B 158 (1979) 141.

[2] D.B. Fairlie, Phys. Lett. B 82 (1979) 97.

[3] D.B. Fairlie, J. Phys. G 5 (1979) L55.

[4] T. Nomura and J. Sato, Nucl. Phys. B 811, 109 (2009) [arXiv:0810.0898 [hep-ph]].

[5] P. Forgacs and N.S. Manton, Commun. Math. Phys. 72 (1980) 15.

[6] D. Kapetanakis and G. Zoupanos, Phys. Rept. 219 (1992) 1. 